\title{
Data Analytics - The ICW System
}

\section{Clare Whalen under the mentorship of Tracy K Lundin \& Albert Eiffes. With support from Emily Knippel, Gregory Gilbert, Paul Ranieri, Pratik Patel, and Beau Harrison.}

\section{The Issue With Current Data Analytic Methods}

Currently at Fermilab, two main systems are being used to collect data on the Industrial Cooling Water (ICW) System. ACNET is used to track the temperature of the water that enters and exits certain buildings. Meanwhile, Metasys keeps tabs on the route the water takes, it's usage, and several other factors.

The problem lies with the type of data analytic method; there are incorrect sensor readings and the data isn't being used to it's full potential. These two systems only go as far as giving the user the ability to find the problem and put a stop to it manually. The goal of data analytics is to predict when, why, and how a negative situation will occur based on the data collected throughout history.

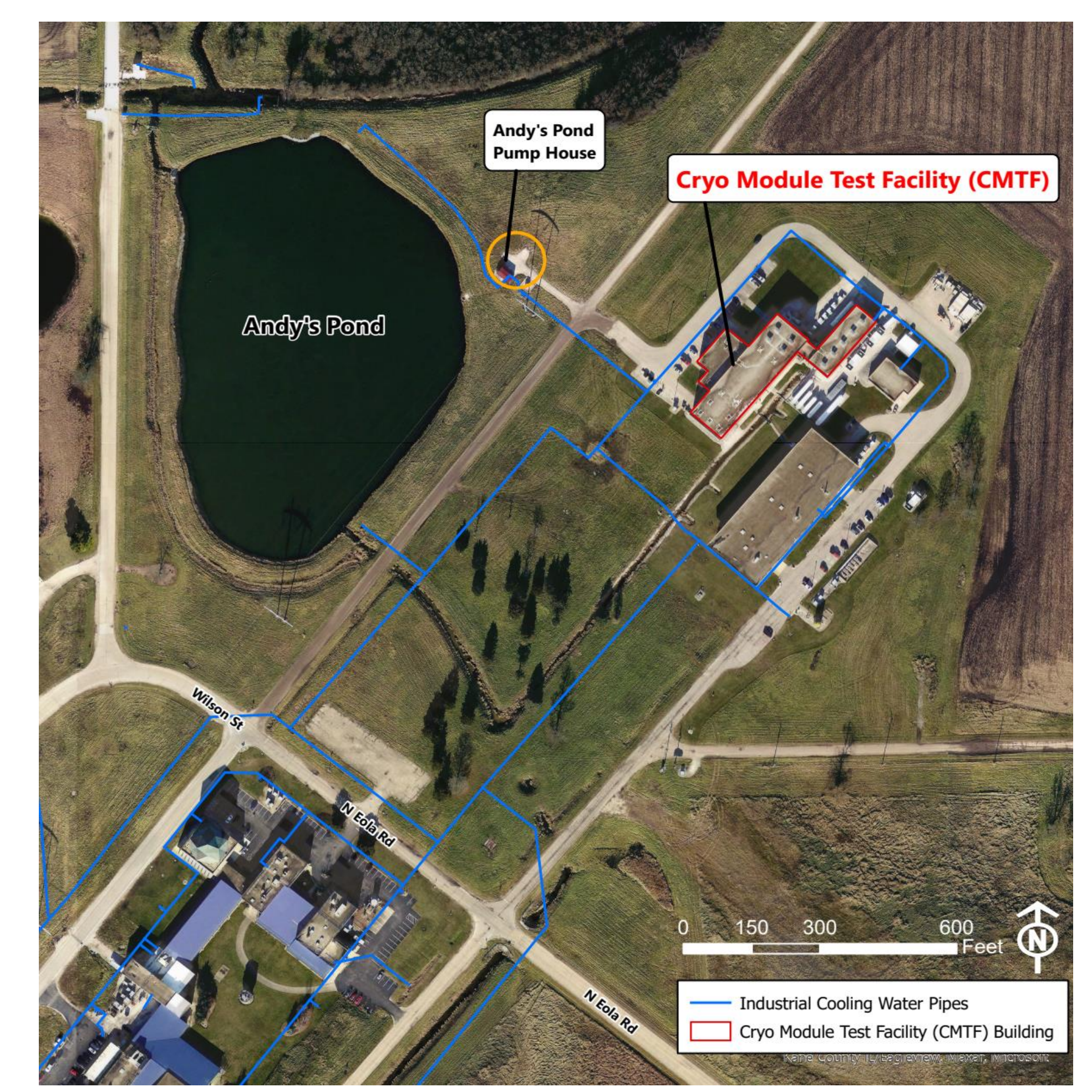

An overview of Andy's Pond, which supplies cooled water to the Cryo Module Test Facility (CMTF) building.

\section{Conclusion}

Based on the data that's been recorded to date in 2021 and the graphs interpreting the data shown on this poster, it is clear that there is a direct relationship between the ambient air temperature in Batavia, IL and the water flow to the CMTF building.

There is a much less obvious relationship between the ICW temperature and the ICW flow. For the most part, the flow of the water going to the CMTF building does increase as the temperature of the Industrial Cooling Water rises. However, there are several outliers that makes this conclusion less certain to be true.

Due to a faulty temperature sensor at the CMTF building, the current graph that compares the ambient temperature and the ICW temperature is inaccurate. This is definitely a problem that needs to be resolved for any type of progression in data analytics to occur. If the data collected is "garbage," then the conclusions will be "garbage."

This is just a small example of what a data analytics program could do in mere seconds. It would greatly benefit Fermilab to run similar tests in order to get to that point of predicting when, why, and how the following events will occur: overflow of water, overheated water, low levels of water, etc.

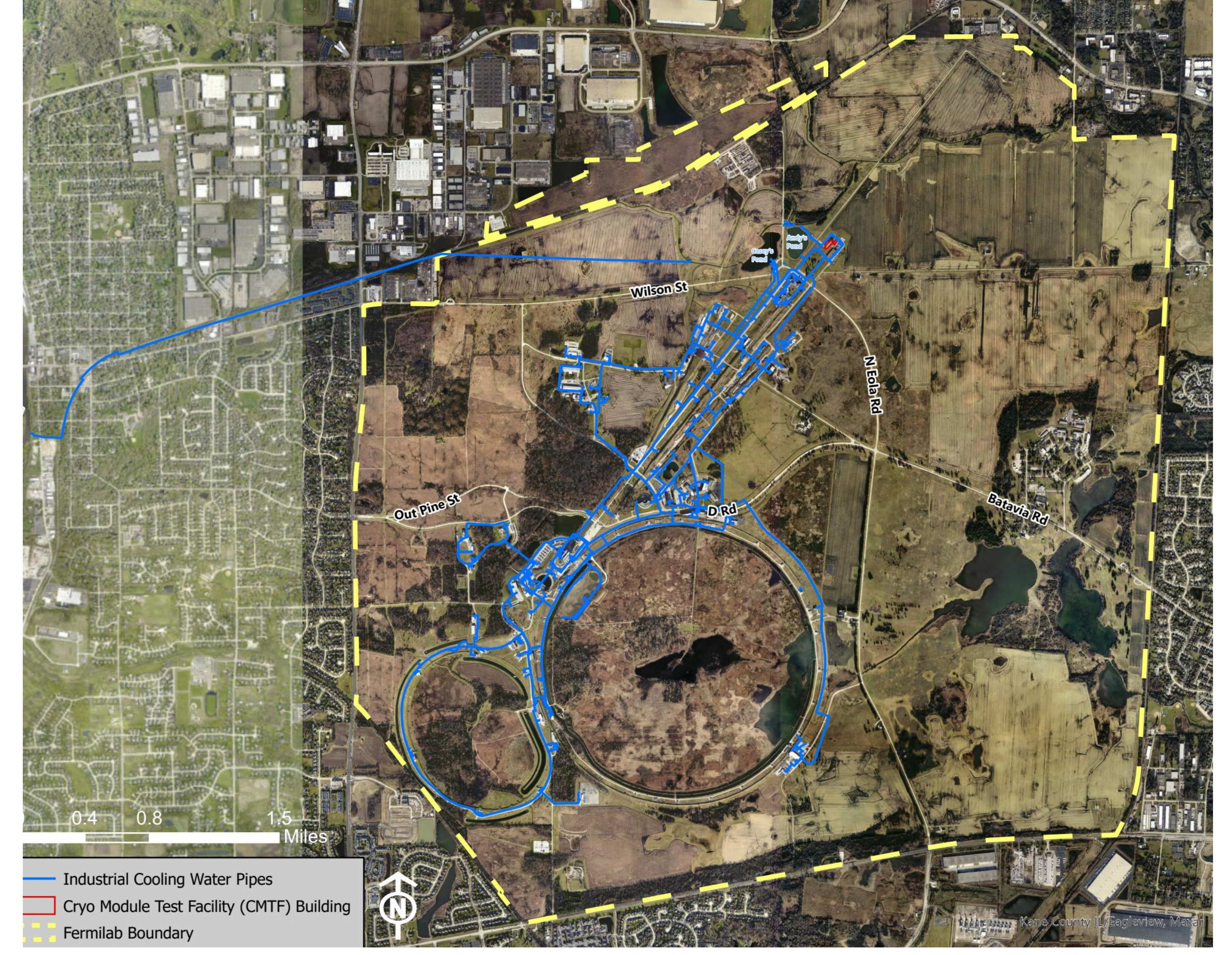

The Industrial Cooling Water (ICW) system Conductivity Water(LCW) system in order to keep the accelerators at a low enough temperature so that they may function properly. The ICW system also supplies water to the fire hydrants and fire protection sprinklers that are onsite. There are five main inputs of water to the system; They are as follows: Casey's Pond Andy's Pond, Swan Andy's Pond, Swan and C4 Pump House.

\section{Hypothesis}

One of the first steps towards predicting when, why, and how something like the Industrial Cooling Water being too hot might occur is finding the relationships between the several variables that are in the system; more specifically, finding the independent and dependent variables.

In this case, a smaller scaled study would do. The purpose of this test was to see if the ambient air temperature in Batavia, IL causes the temperature of the Industrial Cooling Water that travels in and out of the CMTF building to change and therefore increasing or decreasing the flow of water to the same building.

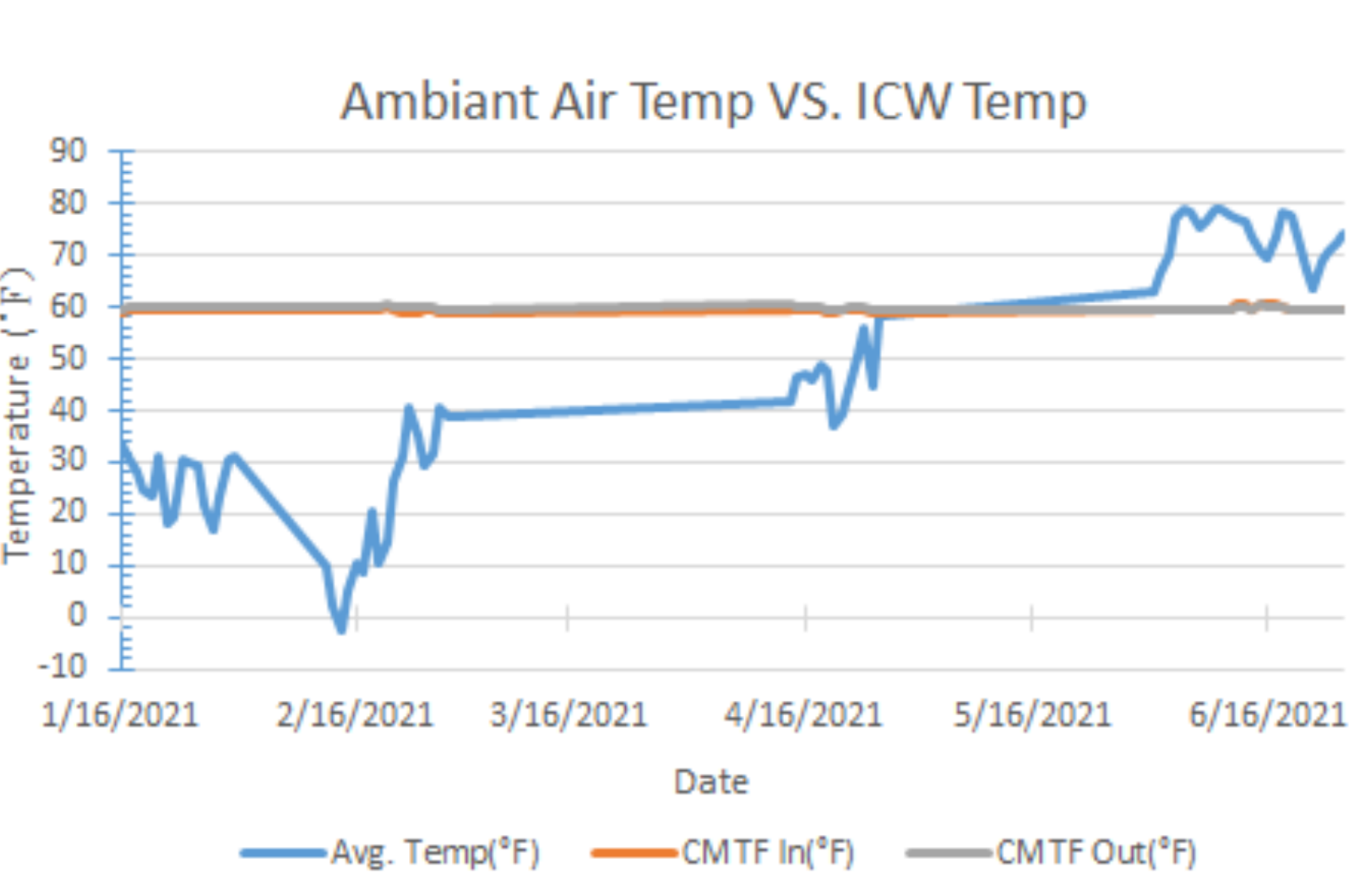

Faulty graph of the ambient air temperature VS the temperature of the Industrial Cooling Water going in and out of the CMTF Building

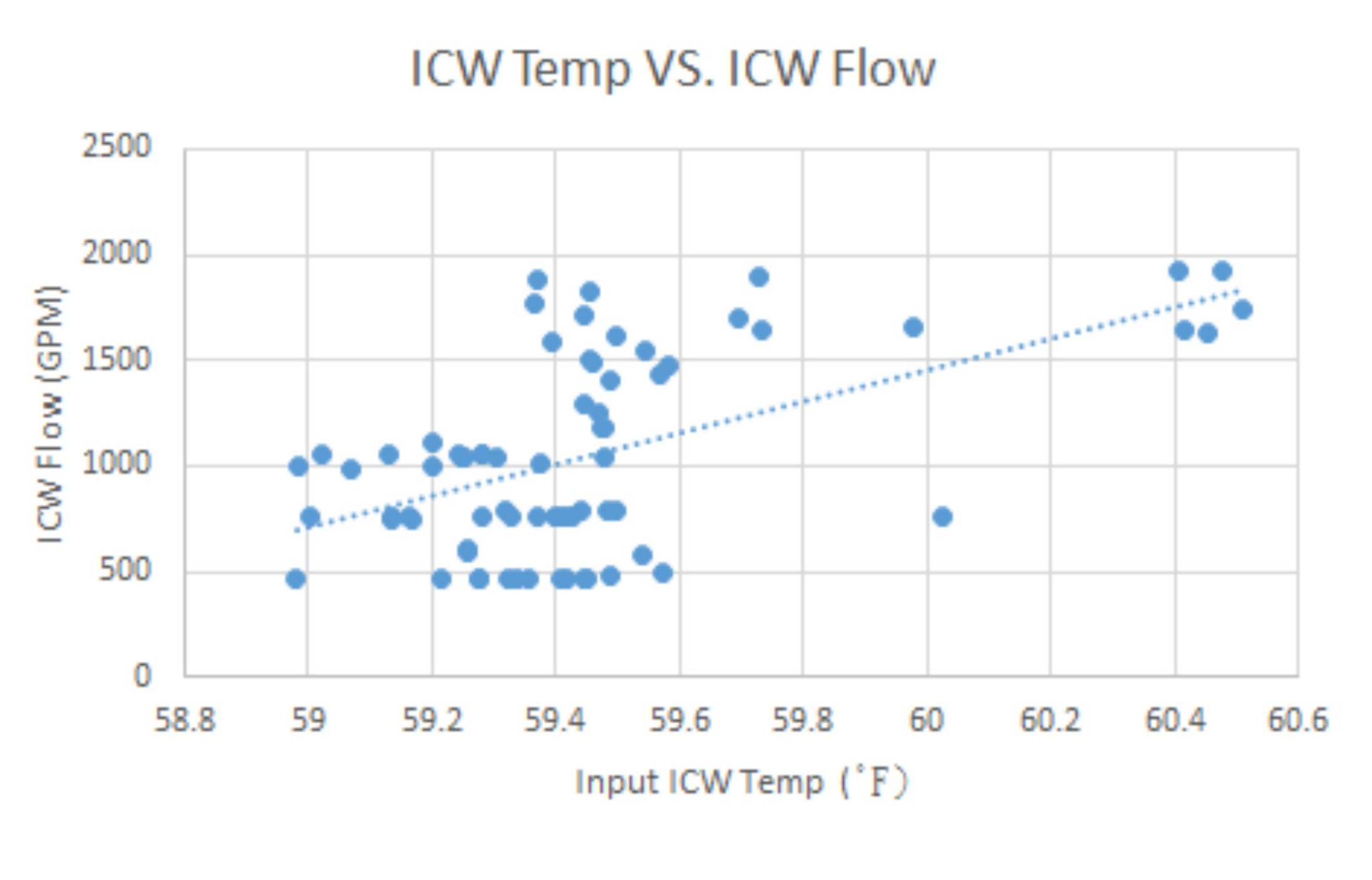

A graph of the temperature of the Industrial cooling water (ICW) going into the CMTF building VS the water flow to the CMTF building.

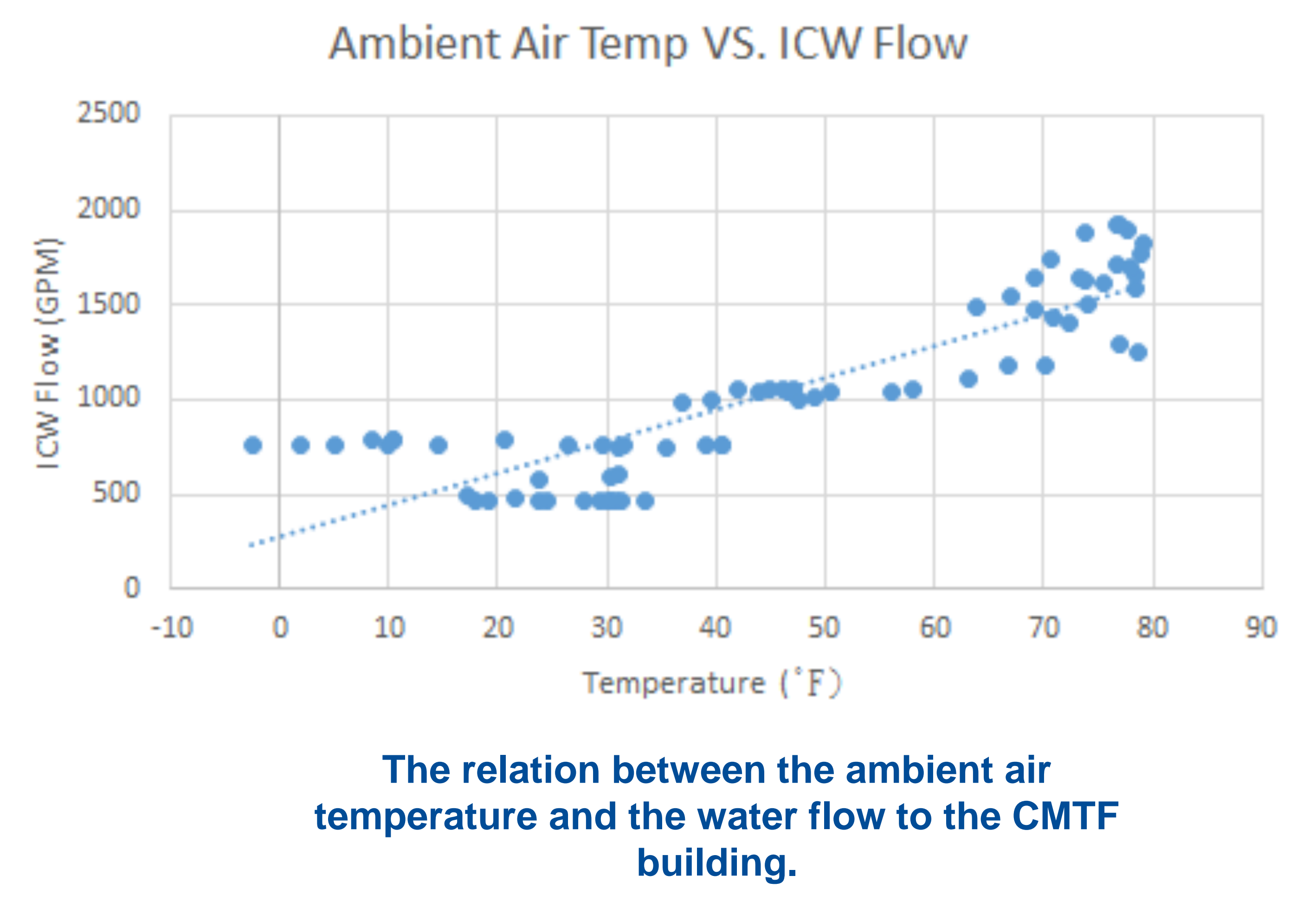

